$11-1-2005$

\title{
JMASM20: Exact Permutation Critical Values For The Kruskal-Wallis One-Way ANOVA
}

Justice I. Odiase

University of Benin, Nigeria

Sunday M. Ogbonmwan

University of Benin, Nigeria

Follow this and additional works at: http://digitalcommons.wayne.edu/jmasm

Part of the Applied Statistics Commons, Social and Behavioral Sciences Commons, and the Statistical Theory Commons

\section{Recommended Citation}

Odiase, Justice I. and Ogbonmwan, Sunday M. (2005) "JMASM20: Exact Permutation Critical Values For The Kruskal-Wallis OneWay ANOVA," Journal of Modern Applied Statistical Methods: Vol. 4 : Iss. 2 , Article 28.

DOI: $10.22237 /$ jmasm/1130804820

Available at: http://digitalcommons.wayne.edu/jmasm/vol4/iss2/28

This Algorithms and Code is brought to you for free and open access by the Open Access Journals at DigitalCommons@WayneState. It has been accepted for inclusion in Journal of Modern Applied Statistical Methods by an authorized editor of DigitalCommons@WayneState. 


\title{
JMASM Algorithms and Code JMASM20: Exact Permutation Critical Values For The Kruskal-Wallis One-Way ANOVA
}

\author{
Justice I. Odiase Sunday M. Ogbonmwan \\ Department of Mathematics \\ University of Benin, Nigeria
}

\begin{abstract}
The exhaustive enumeration of all the permutations of the observations in an experiment is the only possible way of truly constructing exact tests of significance. The permutation paradigm requires no distributional assumptions and works well with values that are normal, almost normal and non-normally distributed. The Kruskal-Wallis test does not require the assumptions that the samples are from normal populations and that the samples have the same standard deviation. In this article, the exact permutation distribution of the Kruskal-Wallis test statistic is generated empirically by actually obtaining all the distinct permutations of an experiment. The tables of exact critical values for the Kruskal-Wallis one-way ANOVA are produced.
\end{abstract}

Keywords: Permutation test, Kruskal-Wallis test, p-value, permutation algorithm, one-way ANOVA.

\section{Introduction}

Variation is inherent in nature and errors are made occasionally when inferences are drawn from experiments. The risk in decision making cannot be totally eliminated but it can be controlled if correct statistical procedures are employed. The unconditional permutation approach is a statistical procedure that ensures that the probability of a type I error is exactly $\alpha$ and ensures that the resulting distribution of the test statistic is exact (Agresti, 1992; Good, 2000; Pesarin, 2001).

Scheffe (1943) demonstrated that for a general class of problems, the permutation approach is the only possible method of

J. I. Odiase is a Lecturer in the Department of Mathematics. His areas of research are statistical computing and nonparametric statistics. Email him at justiceodiase@yahoo.com. S. M. Ogbonmwan is an Associate Professor of Statistics, Department of Mathematics, University of Benin, Nigeria. His areas of research are statistical computing and nonparametric statistics. Email him at ogbonmwasmaltra@yahoo.co.uk. constructing exact tests of significance. It is asymptotically as powerful as the best parametric test (Hoeffding, 1952). In this article, consideration is given to the exhaustive permutation of the ranks of the observations in a single factor multi-sample experiment to arrive at the exact distribution of the Kruskal-Wallis $(\mathrm{K}-\mathrm{W})$ test statistic.

The method of obtaining an exact test of significance originated with Fisher (1935). The essential feature is that all the distinct arrangements of the observations are considered, with the proviso that all permutations are equally likely under the null hypothesis. An exact test on the level of significance $\alpha$ is constructed by choosing a proportion, $\alpha$, of the permutation as the critical region.

Statisticians have considered for some decades the possibility of generating exact critical values for the common test statistics that are in use today. This has resulted in the development of several ways such as the exact conditional permutation approach (Fisher, 1935; Agresti, 1992), the Monte Carlo approaches such as the Bootstrap (Efron, 1979; Efron and Tibshirani, 1993), the Bayesian approach (Casella \& Robert, 2004), and the likelihood approach (Owen, 1988; Barndorff-Nielsen \& Hall, 1988). 
The works of Siegel and Castellan (1989), Conover (1999), Headrick (2003), Bagui $\&$ Bagui (2004) are contributions to the quest for exact critical values but the distributions are obtained from either simulation or asymptotic approximations of the distribution of the $\mathrm{K}-\mathrm{W}$ test statistic. For small samples, $n_{i} \leq 5, i=1(1) p$ in a p-sample experiment, the null distribution of $\mathrm{K}-\mathrm{W}$ statistic is not known and a chi-square approximation will not be a good approximation, (see Bagui \& Bagui (2004)). The consideration given in this article produces the exact distribution of the K-W test statistic for small samples.

Distribution-free analysis of variance

The single-factor ANOVA model for comparing $\mathrm{p}$ populations or treatment means assumes that for $\mathrm{i}=1,2, \ldots, \mathrm{p}$, a random sample of size $\mathrm{n}$ is drawn from a normal population with mean $\mu_{i}$ and variance $\sigma^{2}$. The normality assumption is required for the validity of the $\mathrm{F}$ test while the validity of the Kruskal-Wallis test for testing equality of the $\mu_{\mathrm{i}}$ 's (Kruskal \& Wallis, 1952) depends only on the amount by which observed values deviate from their means $\mu_{\mathrm{i}}$ 's (random error) having the same continuous distribution.

Given a multisample experiment with

$$
X_{i}=\left(X_{i 1}, X_{i 2}, \ldots, X_{i n_{i}}\right)^{T}, \mathrm{i}=1(1) \mathrm{p}
$$

and

$$
\mathbf{X}_{\mathbf{N}}=\left(X_{1}, X_{2}, \ldots, X_{p}\right),
$$

where $\mathrm{N}=\sum_{i=1}^{p} n_{i}$, the total number of observations in the data set. Suppose that one ranks all the $\mathrm{N}$ observations from 1 (smallest $\mathrm{X}_{\mathrm{ij}}$ ) to $\mathrm{N}$ (largest $\mathrm{X}_{\mathrm{ij}}$ ), the permutation test procedure presented in this article, computes an empirical estimate of the cumulative distribution of the test statistic $\mathrm{T}$ under the null hypothesis. Let the layout of the ranks of the observations $\mathrm{X}_{\mathrm{ij}}$ be as follows:

$$
R_{i}=\left(r_{i 1}, r_{i 2}, \ldots, \quad r_{i n_{i}}\right)^{\mathrm{T}}, \mathrm{i}=1(1) \mathrm{p} .
$$

and

$$
\mathbf{R}_{\mathbf{N}}=\left(R_{1}, R_{2}, \ldots, R_{p}\right), \mathrm{N}=\sum_{i=1}^{p} n_{i} .
$$

Under the null hypothesis, $\mathbf{R}_{\mathbf{N}}$ is composed of $\mathrm{N}$ independent and identically distributed random variables and hence conditioned on the observed data set. An exhaustive permutation of the ranks yields

$$
\mathrm{M}=\frac{\boldsymbol{N} !}{\prod_{i=1}^{P}\left[\left(n_{i}\right) !\right]}
$$

permutations of the $\mathrm{N}$ ranks of the variates of $\mathrm{p}$ subsets of size $n_{i}, i=1(1) p$ which are equally likely, each having the conditional probability $\mathrm{M}^{-1}$.

When $H_{0}: \mu_{1}=\mu_{2}=\ldots=\mu_{p}$ is true, the $\mathrm{N}$ observations are assumed to have come from the same distribution, in which case all possible assignments of the rank $1,2, \ldots, \mathrm{N}$ to the $\mathrm{p}$ samples are equally likely and the ranks will be intermingled in these samples. Let $R_{i j}$ denote the rank of the jth observation in the ith treatment $\mathrm{X}_{\mathrm{ij}}$. Let $R_{i}$. and $\bar{R}_{i}$. denote respectively the total and mean of the ranks in the ith treatment. The K-W test statistic is a measure of the extent to which the $\bar{R}_{i}$.'s deviate from their common expected value $\frac{N+1}{2}$, and $\mathrm{H}_{0}$ is rejected if the computed value of the statistic indicates too great a discrepancy between observed and expected rank averages. The K-W test statistic is

$$
\mathrm{H}=\frac{12}{N(N+1)} \sum_{i=1}^{p} \frac{R_{i \bullet}^{2}}{n_{i}}-3(N+1) .
$$

If $\mathrm{H}_{0}$ is rejected when $H \geq c$, then $\mathrm{c}$ should be chosen so that the test has level $\alpha$. That is, c should be the upper-tail critical value of the distribution of $\mathrm{H}$ when $\mathrm{H}_{0}$ is true. Under $\mathrm{H}_{0}$, each possible assignment can be enumerated, the value of $\mathrm{H}$ determined for each one, and the null distribution obtained by 
counting the number of times each value of $\mathrm{H}$ occurs. When $\mathrm{H}_{0}$ is true, the large-sample approximation is applied if $\mathrm{p}=3, n_{i} \geq 6, \mathrm{i}=$ $1(1) 3$ or $p>3, n_{i} \geq 5, \mathrm{i}=1$ (1)p (Devore, 1982; Rohatgi, 1984). $\mathrm{H}$ has approximately a chisquared distribution with $p-1$ degrees of freedom. An approximate level $\alpha$ test is given by: Reject $\mathrm{H}_{0}$ if $H \geq \chi_{\alpha, p-1}^{2}$.

\section{Methodology}

The process of obtaining the permutations starts by choosing the test statistic $\mathrm{T}$ and the acceptable significance level $\alpha$. Let $\pi_{1}, \pi_{2}$, $\ldots, \pi_{\mathrm{n}}$ be a set of all distinct permutations of the ranks of the data set in the experiment. The permutation test procedure is as follows:

1. Rank the observations of the experiment as required by the $\mathrm{K}-\mathrm{W}$ test.

2. Compute the observed value of the K-W test statistic $\left(\mathrm{H}_{1}=\mathrm{t}_{0}\right)$.

3. Obtain a distinct permutation $\pi_{i}$, of the ranks in Step 1.

4. Compute the $\mathrm{K}-\mathrm{W}$ test statistic $\mathrm{H}_{\mathrm{i}}$ for permutation $\pi_{i}$ in Step 3, that is, $\mathrm{H}_{\mathrm{i}}=$ $\mathrm{H}\left(\pi_{i}\right)$.

5. Repeat Steps 3 and 4 for $i=2,3, \ldots, M$.

6. Construct an empirical cumulative distribution for $\mathrm{H}$

$$
p_{0}=\mathrm{p}\left(\mathrm{H} \leq \mathrm{H}_{\mathrm{i}}\right)=\frac{1}{\mathrm{M}} \sum_{\mathrm{i}=1}^{\mathrm{M}} \psi\left(\mathrm{t}_{0}-\mathrm{H}_{\mathrm{i}}\right)
$$

where

$$
\psi(\cdot)=\left\{\begin{array}{ll}
1, & \text { if } \mathrm{t}_{0} \geq H_{\mathrm{i}} \\
0, & \text { if } \mathrm{t}_{0}<H_{\mathrm{i}}
\end{array} .\right.
$$

7.Under the empirical distribution, if $p_{0} \leq \alpha$, reject the null hypothesis.
The complexity in permutation test lies in obtaining all the distinct permutations of the observations in a given experiment. For example, a four-sample experiment with six variates in each sample requires $2,308,743,493,056$ permutations. The frequency distribution is constructed for all the distinct occurrences of the test statistic from which the probability distribution of the test statistic is computed.

The number of permutations of the ranks of a two-sample experiment is

$$
\sum_{i=0}^{n}\left(\begin{array}{c}
n_{1} \\
i
\end{array}\right)\left(\begin{array}{c}
n_{2} \\
i
\end{array}\right), \mathrm{n}=\min \left(\mathrm{n}_{1}, \mathrm{n}_{2}\right),
$$

see Odiase \& Ogbonmwan (2005) for details.

After obtaining the permutations of the ranks of a two sample experiment, the number of ways to permute the ranks of any $n_{3}$ of the combined ranks $\left(\mathrm{n}_{1}+\mathrm{n}_{2}+\mathrm{n}_{3}\right)$ of the variates of the three-sample experiment yields

$$
\left(\begin{array}{c}
n_{1}+n_{2}+n_{3} \\
n_{3}
\end{array}\right) \sum_{i=0}^{n}\left(\begin{array}{c}
n_{1} \\
i
\end{array}\right)\left(\begin{array}{c}
n_{2} \\
i
\end{array}\right)=\left(\begin{array}{c}
\sum_{k=1}^{3} n_{k} \\
n_{3}
\end{array}\right) \sum_{i=0}^{n}\left(\begin{array}{c}
n_{1} \\
i
\end{array}\right)\left(\begin{array}{c}
n_{2} \\
i
\end{array}\right)
$$

A complete enumeration of the distinct permutations of the ranks of a four-sample experiment yields

$$
\left(\begin{array}{c}
\sum_{k=1}^{4} n_{k} \\
n_{4}
\end{array}\right)\left(\begin{array}{c}
\sum_{k=1}^{3} n_{k} \\
n_{3}
\end{array}\right) \sum_{i=0}^{n}\left(\begin{array}{c}
n_{1} \\
i
\end{array}\right)\left(\begin{array}{c}
n_{2} \\
i
\end{array}\right)=\prod_{j=3}^{4}\left(\begin{array}{c}
\sum_{k=1}^{j} n_{k} \\
n_{j}
\end{array}\right) \sum_{i=0}^{n}\left(\begin{array}{c}
n_{1} \\
i
\end{array}\right)\left(\begin{array}{c}
n_{2} \\
i
\end{array}\right)
$$

Continuing in this manner, for $\mathrm{p} \geq 3$ treatments, the distinct permutations of the ranks of the variates are enumerated through

$$
\prod_{j=3}^{p}\left(\begin{array}{c}
\sum_{k=1}^{j} n_{k} \\
n_{j}
\end{array}\right) \sum_{i=0}^{n}\left(\begin{array}{c}
n_{1} \\
i
\end{array}\right)\left(\begin{array}{c}
n_{2} \\
i
\end{array}\right)=\prod_{j=1}^{p}\left(\begin{array}{c}
\sum_{k=1}^{j} n_{k} \\
n_{j}
\end{array}\right) .
$$


For the balanced case, $\mathrm{n}_{1}=\mathrm{n}_{2}=\ldots=\mathrm{n}_{\mathrm{p}}$ $=\mathrm{n}$, the number of distinct permutations of the ranks of the variates is $\prod_{\mathrm{j}=1}^{\mathrm{p}}\left(\begin{array}{c}j n \\ n\end{array}\right)$. As an illustration, let

$$
R_{i}=\left(r_{i 1}, r_{i 2}, \ldots, r_{i n_{i}}\right)^{\mathrm{T}}, \mathrm{i}=1(1) \mathrm{p}
$$

and

$$
\mathbf{R}_{\mathbf{N}}=\left(R_{1}, R_{2}, \ldots, R_{p}\right) .
$$

Consider a three-sample experiment with observations $\mathrm{x}_{\mathrm{ij}}, \mathrm{n}_{1}=3, \mathrm{n}_{2}=\mathrm{n}_{3}=2$, that is, $\left(\begin{array}{lll}x_{11} & x_{21} & x_{31} \\ x_{12} & x_{22} & x_{32} \\ x_{13} & & \end{array}\right)$. Assuming there are no ties, the configuration of the ranks of the experiment can be taken as $\left(\begin{array}{lll}r_{11} & r_{21} & r_{31} \\ r_{12} & r_{22} & r_{32} \\ r_{13} & & \end{array}\right)$. An exhaustive permutation of this experiment yields 210 distinct permutations of the ranks.

First obtain the 6 permutations of the ranks of the 4 variates of the last two treatments, that is,

$$
\begin{aligned}
& \left(\begin{array}{ll}
r_{21} & r_{31} \\
r_{22} & r_{32}
\end{array}\right),\left(\begin{array}{ll}
r_{31} & r_{21} \\
r_{22} & r_{32}
\end{array}\right),\left(\begin{array}{ll}
r_{32} & r_{31} \\
r_{22} & r_{21}
\end{array}\right), \\
& \left(\begin{array}{ll}
r_{21} & r_{22} \\
r_{31} & r_{32}
\end{array}\right),\left(\begin{array}{ll}
r_{21} & r_{31} \\
r_{32} & r_{22}
\end{array}\right),\left(\begin{array}{ll}
r_{31} & r_{21} \\
r_{32} & r_{22}
\end{array}\right),
\end{aligned}
$$

There are 35 ways to permute any 3 ranks of the combined 7 ranks of the variates of the experiment.

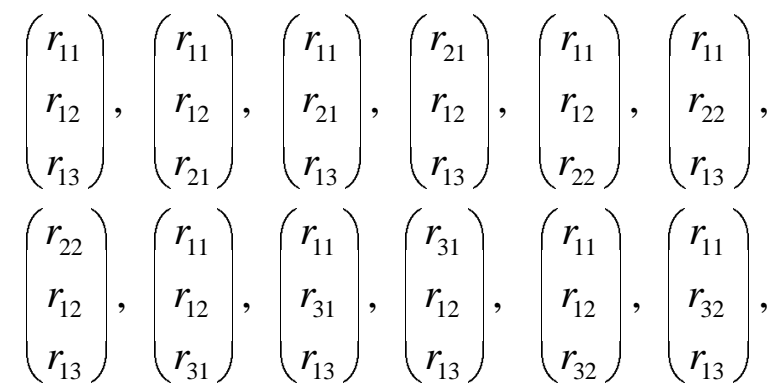

$\left(\begin{array}{l}r_{32} \\ r_{12} \\ r_{13}\end{array}\right),\left(\begin{array}{l}r_{11} \\ r_{21} \\ r_{22}\end{array}\right),\left(\begin{array}{l}r_{21} \\ r_{12} \\ r_{22}\end{array}\right),\left(\begin{array}{l}r_{21} \\ r_{22} \\ r_{13}\end{array}\right),\left(\begin{array}{l}r_{11} \\ r_{21} \\ r_{31}\end{array}\right),\left(\begin{array}{l}r_{21} \\ r_{12} \\ r_{31}\end{array}\right)$, $\left(\begin{array}{l}r_{21} \\ r_{31} \\ r_{13}\end{array}\right),\left(\begin{array}{l}r_{11} \\ r_{21} \\ r_{32}\end{array}\right),\left(\begin{array}{l}r_{21} \\ r_{12} \\ r_{32}\end{array}\right),\left(\begin{array}{l}r_{21} \\ r_{32} \\ r_{13}\end{array}\right),\left(\begin{array}{l}r_{11} \\ r_{22} \\ r_{31}\end{array}\right),\left(\begin{array}{l}r_{22} \\ r_{12} \\ r_{31}\end{array}\right)$, $\left(\begin{array}{l}r_{22} \\ r_{31} \\ r_{13}\end{array}\right),\left(\begin{array}{l}r_{11} \\ r_{22} \\ r_{32}\end{array}\right),\left(\begin{array}{l}r_{22} \\ r_{12} \\ r_{32}\end{array}\right),\left(\begin{array}{l}r_{22} \\ r_{32} \\ r_{13}\end{array}\right),\left(\begin{array}{l}r_{11} \\ r_{31} \\ r_{32}\end{array}\right),\left(\begin{array}{l}r_{31} \\ r_{12} \\ r_{32}\end{array}\right)$, $\left(\begin{array}{l}r_{31} \\ r_{32} \\ r_{13}\end{array}\right),\left(\begin{array}{l}r_{21} \\ r_{22} \\ r_{31}\end{array}\right),\left(\begin{array}{l}r_{21} \\ r_{22} \\ r_{32}\end{array}\right),\left(\begin{array}{l}r_{21} \\ r_{31} \\ r_{32}\end{array}\right),\left(\begin{array}{l}r_{22} \\ r_{31} \\ r_{32}\end{array}\right)$.

Each of the 35 ways will combine with the 6 permutations of the remaining 4 ranks of the variates making up the last two treatments in any configuration of the experiment, that is,

$$
\left(\begin{array}{l}
7 \\
3
\end{array}\right) \sum_{i=0}^{2}\left(\begin{array}{l}
2 \\
i
\end{array}\right)\left(\begin{array}{l}
2 \\
i
\end{array}\right)
$$

Consider the set of all these 210 permutations, for each one of them, compute the test statistic of interest and hence calculate the probability of the different values of the test statistic based on the number of times each is occurring. When ties occur in the data set, the tied observations are usually assigned the mean of the ranks they would have been assigned if they were distinct. Ties do not pose any problem to the permutation test presented in this article. Assuming no ties, the experiment just presented will have ranks $\{1,2,3,4,5,6,7\}$ represented 
as $\left(\begin{array}{lll}1 & 4 & 6 \\ 2 & 5 & 7 \\ 3 & & \end{array}\right)$ and the distinct permutations of

these ranks lead to the remaining 209 permutations.

Permutation algorithms

Considering the associated complexity in a complete enumeration of the distinct permutations necessary for the compilation of the distribution of the $\mathrm{K}-\mathrm{W}$ test statistic, computer algorithms for an exhaustive enumeration are now presented.

The first step in developing permutation algorithm is to formulate an initial configuration of the ranks of the variates of an experiment by taking the trivial configuration given below as:

$$
\left(\begin{array}{ccccc}
1 & n_{1}+1 & n_{1}+n_{2}+1 & \cdots & \sum_{i=1}^{p-1} n_{i}+1 \\
2 & : & : & : & \\
3 & : & : & : & \\
4 & : & : & : & \\
: & : & : & : & \\
: & & & & \\
n_{1} & n_{1}+n_{2} & n_{1}+n_{2}+n_{3} & \cdots & \sum_{i=1}^{p} n_{i}
\end{array}\right)
$$

Algorithm (PERMUTATION) of Odiase \& Ogbonmwan (2005) can handle the permutation of the ranks of the variates in a twosample experiment. Algorithm 1 in this article generates the distinct permutations of the ranks of the variates of a three-sample experiment and relies on the permutation of the ranks of the variates in a two-sample experiment.
Algorithm 2 calls Algorithm 1 and then generates the distinct permutations of the ranks of the variates of a four-sample experiment. Algorithms 1 and 2 can be extended to take care of the sample sizes under consideration.

\section{Results}

Critical values for the K-W test statistic

The algorithms were implemented in Intel Visual Fortran. Figures $1-10$ show the small sample distribution of the K-W test statistic for different sample sizes for 3 and 4 samples. The resulting tables of exact critical values as obtained from the exact permutation distribution of the K-W test statistic are presented in Tables 1 and 2.

\section{Conclusion}

Figures 1 and 2 reveal the fact that the chi squared distribution, which is the large sample approximation of the $\mathrm{K}-\mathrm{W}$ test statistic, will poorly approximate the exact distribution of the $\mathrm{K}-\mathrm{W}$ test statistic for very small sample sizes. As sample sizes increase, the shape of the chi squared distribution begins to emerge as seen in Figures $3-10$.

The critical values for a test statistic are usually determined by cutting off the most extreme $100 \alpha \%$ of the theoretical frequency distribution of the test statistic, where $\alpha$ is the level of significance, see Siegel and Castellan (1989). The critical values of the K-W test statistic contained in Tables 1 and 2 are obtained from the enumeration of all the distinct permutations of the ranks of the variates in an experiment. These critical values are exact and therefore ensures that the probability of a type I error in decisions arising from the use of the K$\mathrm{W}$ test is exactly $\alpha$. 


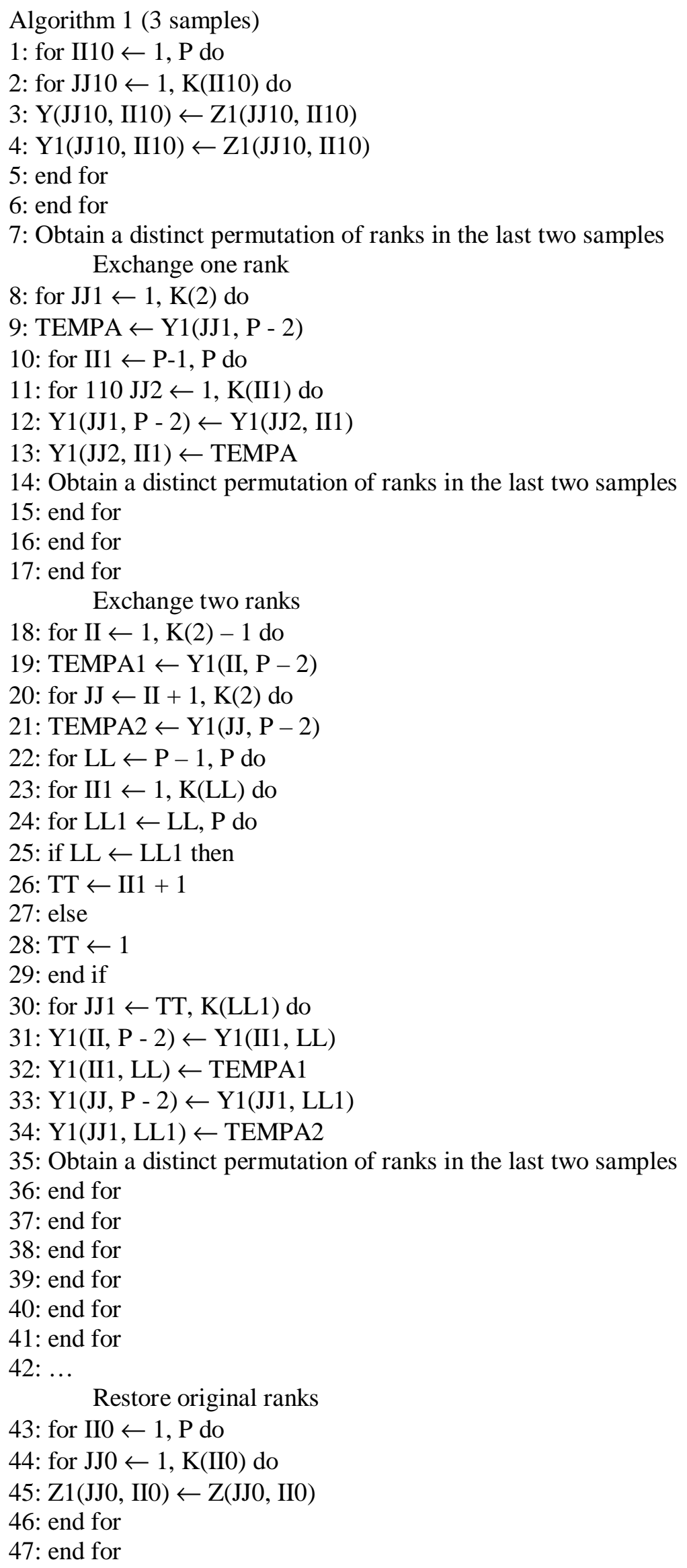




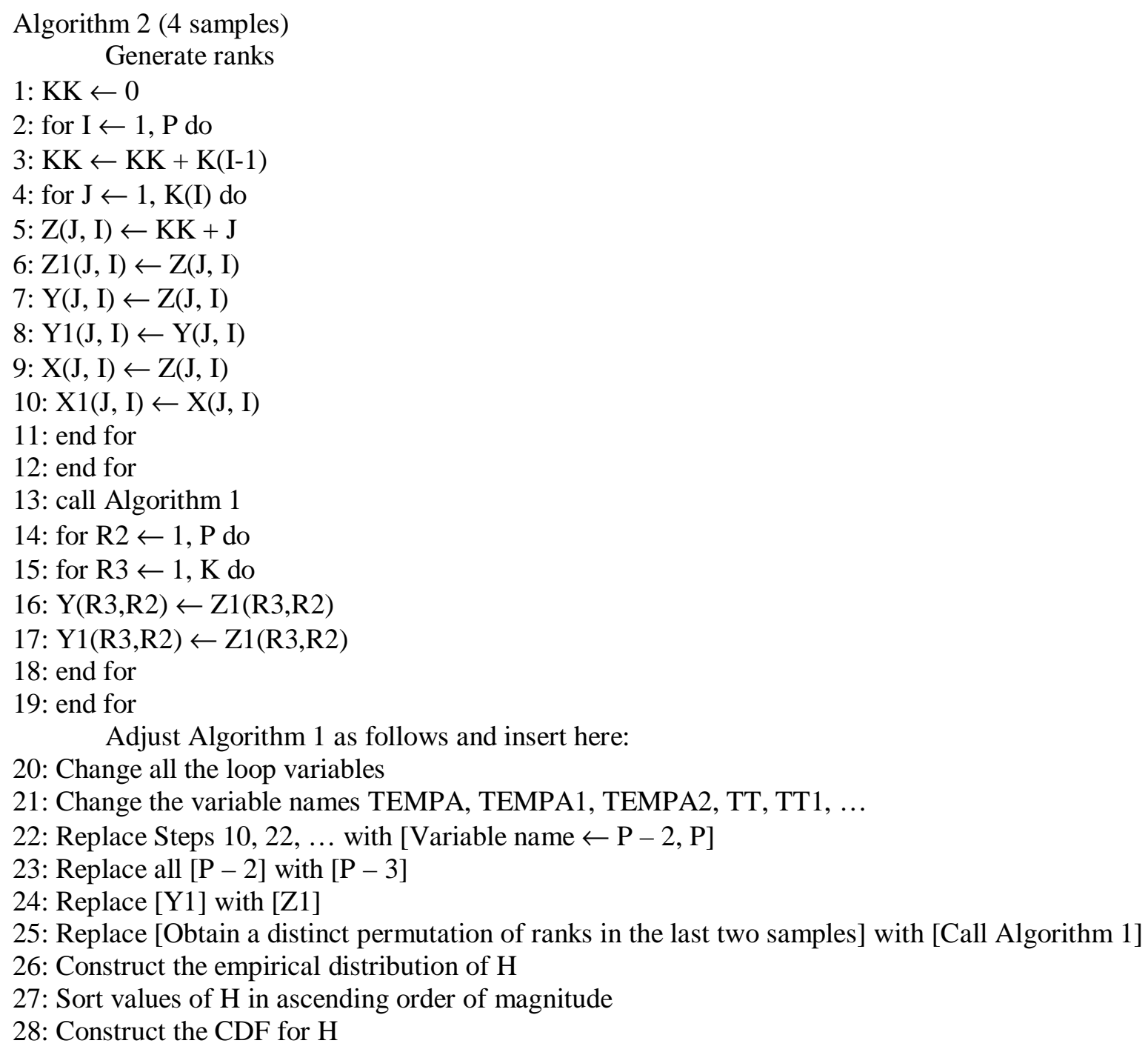


Figures 1 - 10: Distribution of Kruskal-Wallis test statistic for different sample sizes
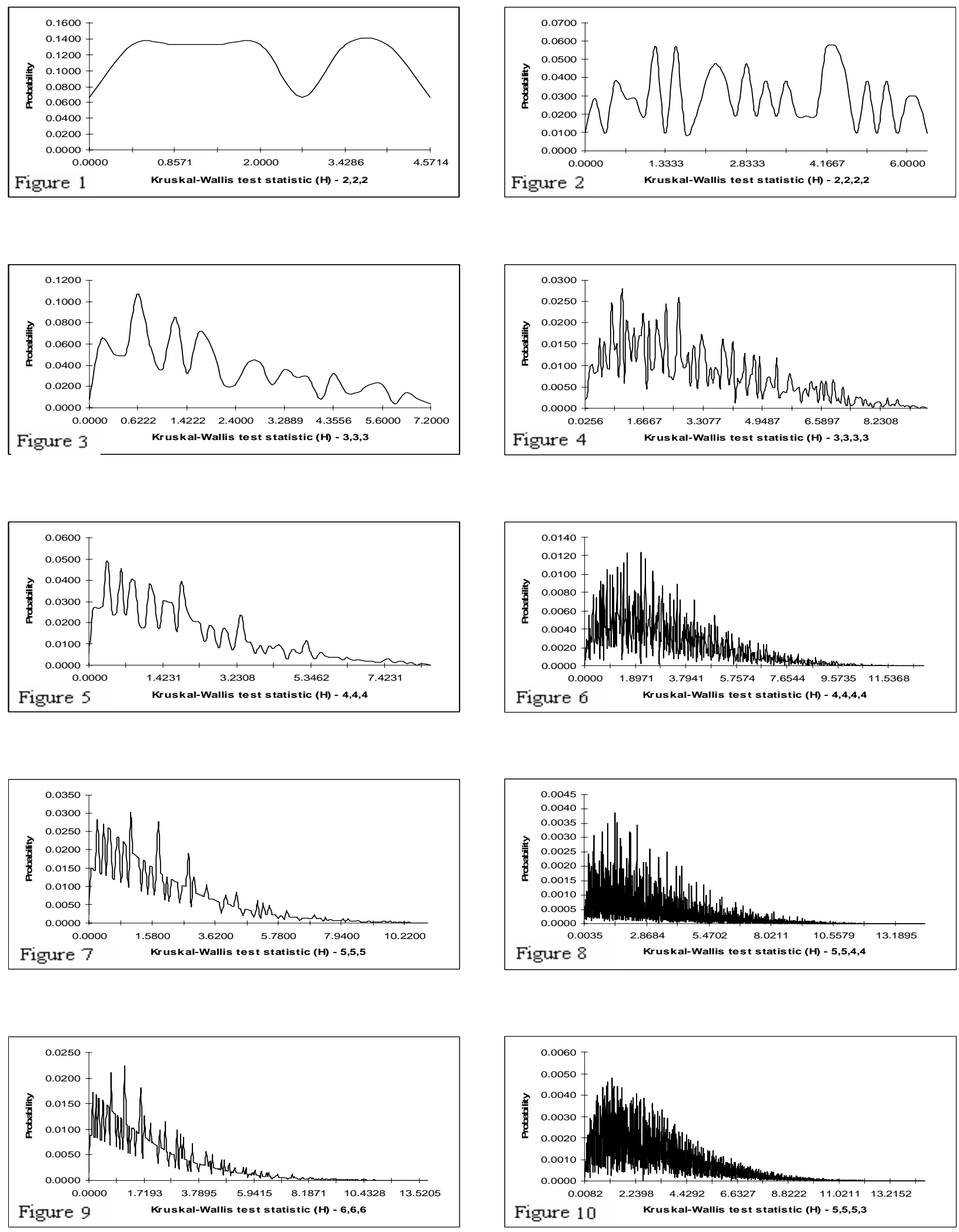
Table 1: Critical values for Kruskal-Wallis test statistic (3 samples)

\begin{tabular}{|c|c|c|c|c|c|c|c|}
\hline Sample Size & $H_{0.9000}$ & $H_{0.9500}$ & $H_{0.9750}$ & $H_{0.9900}$ & $H_{0.9950}$ & $H_{0.9975}$ & $H_{0.9990}$ \\
\hline $2,2,1$ & 3.6000 & & & & & & \\
\hline $2,2,2$ & 3.7143 & 4.5714 & & & & & \\
\hline $3,2,1$ & 4.2857 & & & & & & \\
\hline $3,2,2$ & 4.4643 & 4.5000 & 5.3571 & & & & \\
\hline $3,3,1$ & 4.5714 & & & & & & \\
\hline $3,3,2$ & 4.5556 & 5.1389 & 5.5556 & 6.2500 & & & \\
\hline $3,3,3$ & 4.6222 & 5.6000 & 5.9556 & 6.4889 & & & \\
\hline $4,2,1$ & 4.0179 & 4.8214 & & & & & \\
\hline $4,2,2$ & 4.4583 & 5.1250 & 5.3333 & 6.0000 & & & \\
\hline $4,3,1$ & 3.8889 & 5.4000 & 5.3889 & & & & \\
\hline $4,3,2$ & 4.4444 & 5.4000 & 5.8000 & 6.3000 & 6.4444 & 7.0000 & \\
\hline $4,3,3$ & 4.7000 & 5.7273 & 6.0182 & 6.7455 & 7.0000 & 7.3182 & 8.0182 \\
\hline $4,4,1$ & 4.0667 & 4.8667 & 6.0000 & 6.1667 & & & \\
\hline $4,4,2$ & 4.4455 & 5.2364 & 6.0818 & 6.8727 & 7.0364 & 7.2818 & 7.8545 \\
\hline $4,4,3$ & 4.4773 & 5.5758 & 6.3864 & 7.1364 & 7.4773 & 7.8485 & 8.3258 \\
\hline $4,4,4$ & 4.5000 & 5.6538 & 6.5769 & 7.5385 & 7.7308 & 8.1154 & 8.7692 \\
\hline $5,1,1$ & 3.8571 & & & & & & \\
\hline $5,2,1$ & 4.0500 & 4.4500 & 5.2500 & & & & \\
\hline $5,2,2$ & 4.2933 & 5.0400 & 5.6933 & 6.1333 & & & \\
\hline $5,3,1$ & 3.8400 & 4.8711 & 5.7600 & 6.4000 & & & \\
\hline $5,3,2$ & 4.4945 & 5.1055 & 5.9491 & 6.8218 & 6.9491 & 7.1818 & 7.6364 \\
\hline $5,3,3$ & 4.4121 & 5.5152 & 6.3030 & 6.9818 & 7.5152 & 7.8788 & 8.2424 \\
\hline $5,4,1$ & 3.9600 & 4.8600 & 5.7764 & 6.8400 & 6.9545 & & \\
\hline $5,4,2$ & 4.5182 & 5.2682 & 6.0409 & 7.1182 & 7.5682 & 7.8136 & 8.1136 \\
\hline $5,4,3$ & 4.5231 & 5.6308 & 6.3949 & 7.3949 & 7.9064 & 8.2564 & 8.6256 \\
\hline $5,4,4$ & 4.6187 & 5.6176 & 6.5967 & 7.7440 & 8.1560 & 8.7033 & 9.1286 \\
\hline $5,5,1$ & 4.0364 & 4.9091 & 5.7818 & 6.8364 & 7.7455 & 7.7455 & 8.1818 \\
\hline $5,5,2$ & 4.5077 & 5.2462 & 6.2308 & 7.2692 & 8.0769 & 8.2923 & 8.6846 \\
\hline $5,5,3$ & 4.5363 & 5.6264 & 6.4879 & 7.5429 & 8.2637 & 8.7912 & 9.2835 \\
\hline $5,5,4$ & 4.5200 & 5.6429 & 6.6714 & 7.7914 & 8.4629 & 9.0257 & 9.5057 \\
\hline $5,5,5$ & 4.5000 & 5.6600 & 6.7200 & 7.9800 & 8.7200 & 9.3800 & 9.9200 \\
\hline $6,1,1$ & 4.0833 & & & & & & \\
\hline $6,2,1$ & 3.8222 & 4.6222 & 5.4000 & & & & \\
\hline $6,2,2$ & 4.4364 & 5.0182 & 5.5273 & 6.5455 & 6.6545 & & \\
\hline $6,3,1$ & 3.8182 & 4.8545 & 5.8545 & 6.5818 & & & \\
\hline $6,3,2$ & 4.5455 & 5.2273 & 6.0606 & 6.7273 & 7.5000 & 7.5758 & 8.1818 \\
\hline $6,3,3$ & 4.5385 & 5.5513 & 6.3846 & 7.1923 & 7.6154 & 8.3205 & 8.6282 \\
\hline $6,4,1$ & 3.8636 & 4.9242 & 5.6970 & 7.0833 & 7.5000 & 7.9545 & \\
\hline $6,4,2$ & 4.4359 & 5.2628 & 6.1090 & 7.2115 & 7.8205 & 8.3077 & 8.6667 \\
\hline $6,4,3$ & 4.5989 & 5.6044 & 6.5000 & 7.4670 & 8.0275 & 8.6538 & 9.1703 \\
\hline $6,4,4$ & 4.5238 & 5.6667 & 6.5952 & 7.7238 & 8.3238 & 8.8810 & 9.6286 \\
\hline $6,5,1$ & 3.9205 & 4.8359 & 5.8615 & 6.9974 & 8.0667 & 8.4359 & 8.8846 \\
\hline $6,5,2$ & 4.4747 & 5.3187 & 6.1890 & 7.2989 & 8.1868 & 8.7473 & 9.1890 \\
\hline $6,5,3$ & 4.4971 & 5.6000 & 6.6210 & 7.5600 & 8.2971 & 9.0286 & 9.6686 \\
\hline $6,5,4$ & 4.5000 & 5.6558 & 6.7358 & 7.8958 & 8.6400 & 9.2933 & 9.9600 \\
\hline $6,5,5$ & 4.5294 & 5.6985 & 6.7809 & 8.0118 & 8.8353 & 9.5809 & 10.2706 \\
\hline $6,6,1$ & 3.9780 & 4.8571 & 5.9121 & 7.0659 & 7.9341 & 8.9231 & 9.3077 \\
\hline $6,6,2$ & 4.4190 & 5.3524 & 6.1714 & 7.4095 & 8.1524 & 8.9333 & 9.6762 \\
\hline $6,6,3$ & 4.5250 & 5.6000 & 6.6833 & 7.6833 & 8.4167 & 9.2250 & 10.1250 \\
\hline $6,6,4$ & 4.5184 & 5.7206 & 6.7831 & 7.9890 & 8.7206 & 9.4118 & 10.3419 \\
\hline $6,6,5$ & 4.5412 & 5.7516 & 6.8379 & 8.1190 & 8.9817 & 9.7242 & 10.5242 \\
\hline $6,6,6$ & 4.5380 & 5.7193 & 6.8772 & 8.1871 & 9.0877 & 9.8713 & 10.8421 \\
\hline
\end{tabular}


618 EXACT PERMUTATION CRITICAL VALUES FOR THE KRUSKAL-WALLIS

Table 2: Critical values for Kruskal-Wallis test statistic (4 samples)

\begin{tabular}{|c|c|c|c|c|c|c|c|}
\hline Sample Size & $H_{0.9000}$ & $H_{0.9500}$ & $H_{0.9750}$ & $H_{0.9900}$ & $H_{0.9950}$ & $H_{0.9975}$ & $H_{0.9990}$ \\
\hline $2,2,1,1$ & 4.7143 & & & & & & \\
\hline $2,2,2,1$ & 5.0357 & 5.3571 & 5.6786 & & & & \\
\hline $2,2,2,2$ & 5.5000 & 6.0000 & 6.1667 & & & & \\
\hline $3,2,1,1$ & 4.8929 & 5.4643 & & & & & \\
\hline $3,2,2,1$ & 5.3889 & 5.8056 & 6.0556 & 6.5000 & & & \\
\hline $3,2,2,2$ & 5.6444 & 6.2444 & 6.6444 & 7.0000 & 7.1333 & 7.5333 & \\
\hline $3,3,1,1$ & 5.2222 & 5.8889 & & & & & \\
\hline $3,3,2,1$ & 5.6222 & 6.1556 & 6.5111 & 7.0444 & 7.2000 & 7.4000 & \\
\hline $3,3,2,2$ & 5.7273 & 6.4727 & 7.0000 & 7.6364 & 7.7273 & 8.0000 & 8.1273 \\
\hline $3,3,3,1$ & 5.5818 & 6.5273 & 6.8909 & 7.3273 & 7.7636 & 8.0545 & 8.3455 \\
\hline $3,3,3,2$ & 5.8182 & 6.6818 & 7.4697 & 7.9545 & 8.3182 & 8.5606 & 8.9242 \\
\hline $3,3,3,3$ & 5.9744 & 6.8974 & 7.6154 & 8.4359 & 8.7436 & 9.1538 & 9.4615 \\
\hline $4,2,1,1$ & 5.2083 & 5.4583 & 6.0833 & & & & \\
\hline $4,2,2,1$ & 5.5000 & 6.0000 & 6.5000 & 6.8000 & & & \\
\hline $4,2,2,2$ & 5.6727 & 6.4364 & 6.9818 & 7.3091 & 7.8545 & 7.9636 & 8.2909 \\
\hline $4,3,1,1$ & 4.9778 & 6.0444 & 6.5667 & 6.7111 & & & \\
\hline $4,3,2,1$ & 5.5727 & 6.3000 & 6.9091 & 7.3636 & 7.7273 & 7.8909 & 8.1818 \\
\hline $4,3,2,2$ & 5.7121 & 6.6136 & 7.3182 & 7.8485 & 8.2500 & 8.5909 & 8.8939 \\
\hline $4,3,3,1$ & 5.6667 & 6.5379 & 7.2727 & 7.7500 & 8.1212 & 8.3561 & 8.8409 \\
\hline $4,3,3,2$ & 5.8590 & 6.7821 & 7.5577 & 8.3205 & 8.7179 & 9.0577 & 9.4038 \\
\hline $4,3,3,3$ & 6.0000 & 6.9670 & 7.7582 & 8.6538 & 9.2308 & 9.5769 & 10.0000 \\
\hline $4,4,1,1$ & 5.1273 & 5.8636 & 6.9273 & 7.5000 & & & \\
\hline $4,4,2,1$ & 5.5455 & 6.3636 & 7.1364 & 7.8864 & 8.2273 & 8.5682 & 8.7045 \\
\hline $4,4,2,2$ & 5.7692 & 6.6923 & 7.5192 & 8.3077 & 8.6731 & 9.0577 & 9.4423 \\
\hline $4,4,3,1$ & 5.6603 & 6.6154 & 7.4808 & 8.2179 & 8.5769 & 8.8654 & 9.2949 \\
\hline $4,4,3,2$ & 5.8901 & 6.8626 & 7.7363 & 8.6099 & 9.1538 & 9.4835 & 9.9121 \\
\hline $4,4,3,3$ & 6.0048 & 7.0333 & 7.9238 & 8.8667 & 9.4905 & 9.9667 & 10.4619 \\
\hline $4,4,4,1$ & 5.6374 & 6.7088 & 7.6319 & 8.5714 & 8.9505 & 9.2473 & 9.7253 \\
\hline $4,4,4,2$ & 5.9000 & 6.9429 & 7.8857 & 8.8571 & 9.4714 & 9.9143 & 10.4000 \\
\hline $4,4,4,3$ & 6.0292 & 7.1292 & 8.0542 & 9.0667 & 9.7167 & 10.3417 & 10.9000 \\
\hline $4,4,4,4$ & 6.0662 & 7.2132 & 8.2059 & 9.2647 & 9.9485 & 10.5662 & 11.3382 \\
\hline $5,2,1,1$ & 5.1067 & 5.7600 & 6.0667 & 6.6000 & & & \\
\hline $5,2,2,1$ & 5.5309 & 6.0327 & 6.5782 & 7.2000 & 7.4727 & 7.8000 & \\
\hline $5,2,2,2$ & 5.6182 & 6.5273 & 7.1545 & 7.6636 & 8.0182 & 8.3818 & 8.6818 \\
\hline $5,3,1,1$ & 5.1309 & 6.0036 & 6.8764 & 7.1673 & 7.4000 & & \\
\hline $5,3,2,1$ & 5.5030 & 6.3303 & 7.0939 & 7.7455 & 8.1818 & 8.2909 & 8.7273 \\
\hline $5,3,2,2$ & 5.7538 & 6.6564 & 7.4641 & 8.1949 & 8.6256 & 8.9333 & 9.4231 \\
\hline $5,3,3,1$ & 5.6564 & 6.6000 & 7.4205 & 8.1179 & 8.5282 & 8.8974 & 9.2564 \\
\hline $5,3,3,2$ & 5.8571 & 6.8220 & 7.6505 & 8.5912 & 9.0571 & 9.4176 & 9.8549 \\
\hline $5,3,3,3$ & 5.9981 & 7.0114 & 7.8267 & 8.8400 & 9.4571 & 9.9067 & 10.4095 \\
\hline $5,4,1,1$ & 5.2000 & 6.0182 & 6.8000 & 7.8591 & 8.2000 & 8.2955 & 8.6364 \\
\hline $5,4,2,1$ & 5.5615 & 6.4077 & 7.2115 & 8.1692 & 8.5731 & 8.9423 & 9.3231 \\
\hline $5,4,2,2$ & 5.7725 & 6.7220 & 7.5989 & 8.4692 & 9.0495 & 9.4451 & 9.8604 \\
\hline $5,4,3,1$ & 5.6396 & 6.6813 & 7.5253 & 8.3989 & 8.9802 & 9.3484 & 9.7934 \\
\hline $5,4,3,2$ & 5.8933 & 6.9171 & 7.7933 & 8.8000 & 9.3933 & 9.8733 & 10.3543 \\
\hline $5,4,3,3$ & 6.0292 & 7.0892 & 7.9892 & 9.0292 & 9.6958 & 10.2892 & 10.8558 \\
\hline $5,4,4,1$ & 5.6686 & 6.7429 & 7.6743 & 8.7171 & 9.3029 & 9.6971 & 10.2114 \\
\hline $5,4,4,2$ & 5.9400 & 6.9850 & 7.9475 & 9.0000 & 9.6625 & 10.2525 & 10.7875 \\
\hline $5,4,4,3$ & 6.0346 & 7.1669 & 8.1346 & 9.2118 & 9.9397 & 10.5574 & 11.2963 \\
\hline $5,4,4,4$ & 6.0608 & 7.2569 & 8.2725 & 9.3902 & 10.1373 & 10.8020 & 11.5882 \\
\hline $5,5,1,1$ & 5.0923 & 6.0154 & 6.8769 & 8.0769 & 8.6000 & 8.9077 & 9.0923 \\
\hline
\end{tabular}


Table 2: Continued

\begin{tabular}{l|rrrrrrr}
\hline \multirow{2}{*}{ Sample Size } & $H_{0.9000}$ & $H_{0.9500}$ & $H_{0.9750}$ & $H_{0.9900}$ & $H_{0.9950}$ & $H_{0.9975}$ & $H_{0.9990}$ \\
& & & & & & & \\
\hline $5,5,2,1$ & 5.5648 & 6.5341 & 7.2725 & 8.3077 & 9.0198 & 9.4352 & 9.7582 \\
$5,5,2,2$ & 5.7943 & 6.7714 & 7.6457 & 8.6286 & 9.2914 & 9.8800 & 10.3429 \\
$5,5,3,1$ & 5.6476 & 6.7371 & 7.6286 & 8.5962 & 9.2743 & 9.7619 & 10.2191 \\
$5,5,3,2$ & 5.9150 & 6.9417 & 7.8750 & 8.9467 & 9.6350 & 10.1667 & 10.8200 \\
$5,5,3,3$ & 6.0118 & 7.1176 & 8.0588 & 9.1882 & 9.9176 & 10.5529 & 11.2353 \\
$5,5,4,1$ & 5.6625 & 6.7800 & 7.7625 & 8.8625 & 9.5500 & 10.1025 & 10.5900 \\
$5,5,4,2$ & 5.9338 & 7.0279 & 8.0162 & 9.1500 & 9.8868 & 10.5154 & 11.1904 \\
$5,5,4,3$ & 6.0523 & 7.2157 & 8.2092 & 9.3562 & 10.1307 & 10.7895 & 11.5739 \\
$5,5,4,4$ & 6.0684 & 7.2895 & 8.3421 & 9.5351 & 10.3281 & 11.0228 & 11.8439 \\
$5,5,5,1$ & 5.6824 & 6.8294 & 7.8000 & 9.0176 & 9.7588 & 10.3941 & 10.9588 \\
$5,5,5,2$ & 5.9451 & 7.0745 & 8.0941 & 9.2863 & 10.0980 & 10.7451 & 11.5137 \\
$5,5,5,3$ & 6.0433 & 7.2456 & 8.2889 & 9.4959 & 10.3193 & 10.9930 & 11.8257 \\
& & & & & & & \\
\hline
\end{tabular}

\section{References}

Agresti, A. (1992). A survey of exact inference for contingency tables, Statistical Science, 7, 131-177.

Bagui, S., \& Bagui, S. (2004). An algorithm and code for computing exact critical values for the Kruskal-Wallis nonparametric one-way ANOVA. Journal of Modern Applied Statistical Methods, 3, 498-503.

Barndorff-Nielsen, O. E., \& Hall, P. (1988). On the level-error after Bartlett adjustment of the likelihood ratio statistic. Biometrika, 75, 374-378.

Conover, W. J. (1999). Practical nonparametric statistics. New York: Wiley.

Devore, J. L. (1982). Probability and statistics for engineering and the sciences. California: Brooks/Cole Publishing Company.

Efron, B. (1979). Bootstrap methods: Another look at the jackknife. The Annals of Statistics, 7, 1-26.

Efron, B., \& Tibshirani, R. J. (1993). An introduction to the bootstrap. New York: Chapman and Hall.
Fisher, R. A. (1935). The design of experiments. Edinburgh: Oliver and Boyd

Good, P. (2000). Permutation tests: A practical guide to resampling methods for testing hypotheses (2nd ed.). New York: Springer Verlag.

Headrick, T. C. (2003). An algorithm for generating exact critical values for the Kruskal-Wallis one-way ANOVA. Journal of Modern Applied Statistical Methods, 2, 268271.

Hoeffding, W. (1952). Large sample power of tests based on permutations of observations. The Annals of Mathematical Statistics, 23, 169-192.

Kruskal, W. H., \& Wallis, W. A. (1952). Use of ranks in one-criterion variance analysis. Journal of the American Statistical Association, 47, 583-634.

Odiase, J. I., \& Ogbonmwan, S. M. (2005) An algorithm for generating unconditional exact permutation distribution for a two-sample experiment. Journal of Modern Applied Statistical Methods, 4, 319-332. 
Owen, A. B. (1988). Empirical likelihood ratio confidence intervals for a single functional. Biometrika, 75, 237-249.

Pesarin, F. (2001). Multivariate permutation tests. New York: Wiley.

Rohatgi, V. K. (1984). Statistical inference. New York: John Wiley \& Sons.
Scheffe, H. (1943) Statistical inference in the nonparametric case. The Annals of Mathematical Statistics, 14, 305-332.

Siegel, S., \& Castellan, N. J. (1989). Nonparametric statistics for the behavioural sciences (3rd ed.). New York: McGraw-Hill. 